\title{
Tomographic Analysis of EmaA Adhesin Glycosylation in Aggregatibacter actinomycetemcomitans
}

\author{
Alison Watson ${ }^{1}$, Hannah Naughton ${ }^{1}$, Michael Radermacher ${ }^{1}$, Keith P. Mintz $^{2}$, and Teresa Ruiz ${ }^{1}$ \\ ${ }^{1}$ Departments of Molecular Physiology \& Biophysics, University of Vermont, Burlington, VT \\ ${ }^{2}$ Microbiology \& Molecular Genetics, University of Vermont, Burlington, VT
}

The numbers of antibiotic-resistant bacteria has rapidly increased in recent years, thus current research is directed at developing new therapies targeting colonization. Host adherence is frequently considered the first step in the infectious process and is crucial for colonization. A. actinomycetemcomitans is a Gram bacterium involved in periodontal diseases and other systemic infections (e.g. pneumonia, and endocarditis). These infections are associated with numerous virulence determinants, including extracellular matrix adhesins. EmaA (extracellular matrix protein adhesin A), a trimeric autotransporter adhesin, mediates the binding of this bacterium to collagen. EmaA forms antennae-like structures that protrude from the bacterial surface. The $30 \mathrm{~nm}$ distal region of the structure, which is further divided into three subdomains (SI, SII, SIII), contains the N-terminal sequences of the three monomers and encompasses the collagen binding activity [1]. Deletion of this region or any of the subdomains abrogates collagen binding. Furthermore, EmaA is glycosylated, and this post-translational modification is essential for the function of adhesin. Interestingly, this glycoprotein uses a similar synthesis pathway as the O-polysaccharide (O-PS) synthesis of lipopolysaccharides (LPS). Disabling sugar synthesis, transport, and glycan ligation in this pathway changes EmaA function [2]. Yet, the link between the modifications of the protein by glycosylation remains unclear.

The aim of this study is to analyze the 3D structure of the functional domain of the EmaA adhesin from a mutant strain with a disrupted glycosylation mechanism. The waaL mutant strain lacks the O-antigen ligase, WaaL, which is an essential component of the O-PS glycosylation pathway. Structural comparison of the glycosylated and non-glycosylated adhesins will help to determine the structural role of this modification in collagen binding. Bacteria, grown as previously described [3], were adsorbed onto hydrophilic carbon-coated grids that had been pretreated with a colloidal gold solution (fiducial markers) and were negatively stained with Nano-W. Tomographic single-axis tilt series $(\sim 50)$ were acquired over a $\pm 64^{\circ}$ angular range in $2^{\circ}$ intervals using a Tecnai12 electron microscope at $42000 \mathrm{x}$ nominal magnification, which corresponds to a calibrated $3.08 \AA$ pixel at the specimen scale (Fig. 1A). Tilt series were processed using IMOD [4] to generate tomograms (Fig. 1B). Individual EmaA adhesins $(>30)$ were selected for further processing. Reconstructions of EmaA adhesin subvolumes (Fig. 2) were calculated using EMIRA and visualized in Chimera [5,6]. Subvolumes were low-pass filtered and aligned to a reference volume of the wild type EmaA adhesin (Fig. 2, center). Alignment parameters were transferred and applied to each of the unfiltered subvolumes and an average was calculated. This process was repeated multiple times to refine the translational and rotational shift parameters. Aligned subvolumes were averaged together and compared to the wild type EmaA volume (Fig. 2, right).

We observed that the waaL mutant cells produced fewer EmaA adhesins than the wild type cells. This is consistent with what has been previously described [2], and indicates that glycosylation of EmaA may be significant for the stability, as well as function of the adhesin. Glycosilation, however, does not seem to affect the characteristic bending pattern of the antennae-like structures. Based on the present analysis, the structure of the EmaA adhesins present in the waaL mutant A. actinomycetemcomitans strain does 
not appear to be significantly different from wild type. The functional region maintains the complex architecture composed of 3 subdomains (SI-SIII). SI has a globular shape $(\varnothing=5 \mathrm{~nm})$, while SII and SIII have a cylindrical shape and are separated by a linker region of $3 \mathrm{~nm}$ in length. However, there appears to be a subtle bend between the SI-SII regions (Fig. 2, right). This bend is reminiscent of structural changes observed in a different the G162S substitution mutant strain that exhibits greatly reduced collagen binding activity [3]. Currently, we are increasing the number of subvolumes in the data set to resolve the structure at higher resolution to better evaluate the significance of this potential structural difference.

\section{References:}

[1] Yu, C., Mintz, K.P., Ruiz, T., J. Bacteriol. 191 (2009), p.6253.

[2] Tang, G., Ruiz, T., Mintz, K.P., Infect. Immun. 80 (2012), p.2868.

[3] Azari, F., Radermacher, M., Mintz, K.P., Ruiz, T., J. Struct. Biol. 177 (2011), p.439.

[4] Kremer, J.R., Mastronarde, D.N., McIntosh, J.R., J. Struct. Biol. 116 (1996), p.71.

[5] M. Radermacher, Micoscopy \& Microanalysis, 19 (2013), Supplement S2 p.762.

[6] Petterson, E.F., Goddard, T.D., Huang, C.C., et al., J. Comput. Chem. 25 (2004), p.1605.

[7] This work was supported by NIH grant DE024554, (T.R. \& K.P.M) and benefitted from developments from NIH grant GM078202 (M.R.).
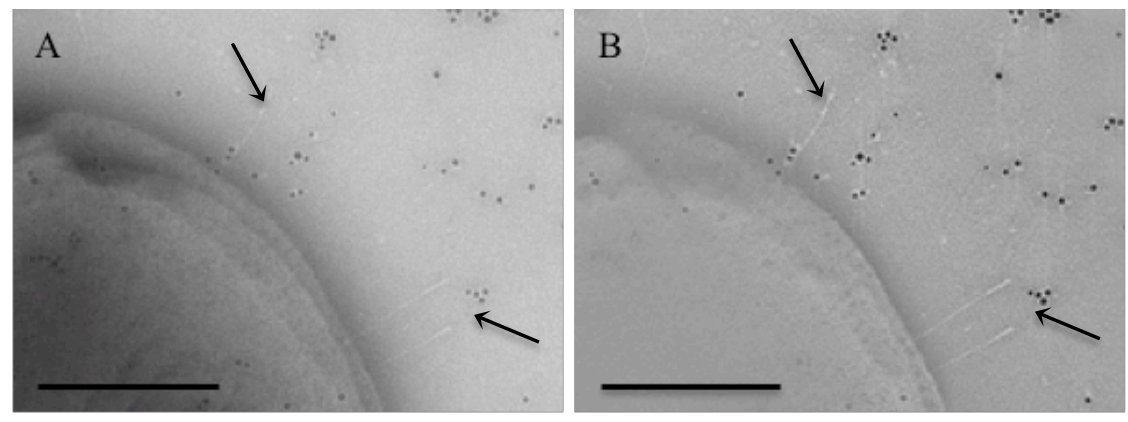

Figure 1. Electron tomography of the waaL mutant bacteria: A) $0^{\circ}$ projection of the tilt-series; B) projection of the central slices of the reconstructed tomogram. Arrows point to EmaA. Bar $=150 \mathrm{~nm}$.

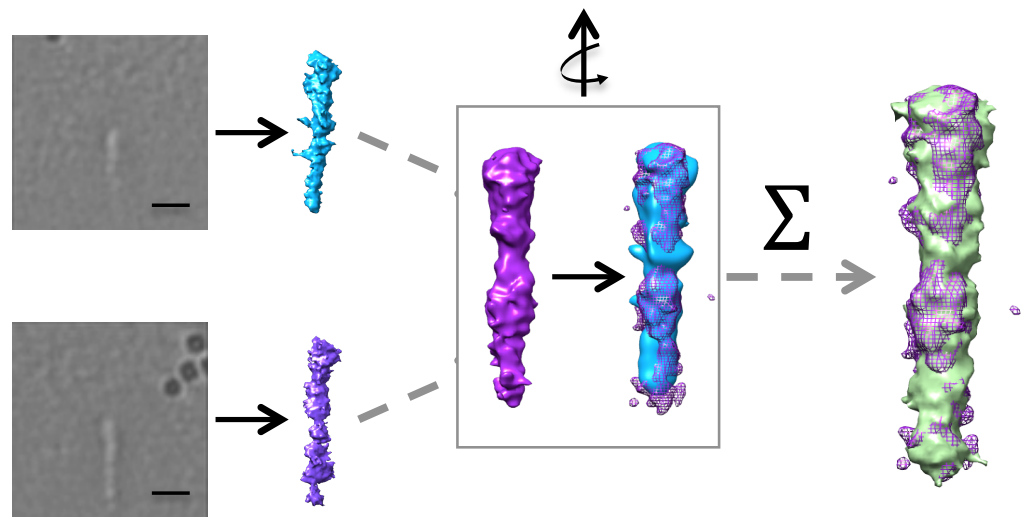

Figure 2. Alignment and averaging of waaL mutant bacteria EmaA subvolumes extracted from tomograms: left) $0^{\circ}$ projection and surface representation of two adhesin subvolumes; center) depiction of alignment procedure to the wild type reference subvolume (purple, [1]); right) average of selected subvolumes. Reference volume is shown as a purple mesh overlay. Bar $=10 \mathrm{~nm}$. 